\title{
High-Temperature Superconductivity
}

\author{
John D. Dow ${ }^{1}$ and Dale R. Harshman ${ }^{1,2}$ \\ ${ }^{1}$ Department of Physics, Arizona State University, Tempe, Arizona 85287 U.S.A. \\ ${ }^{2}$ Physikon Research Corporation, P. O. Box 1014, Lynden, WA 98264 U.S.A.*
}

Received on 23 May, 2003.

\begin{abstract}
The widely held notion that high-temperature superconductivity originates in the cuprate-planes is proven to be faulty. In the cuprates such as $\mathrm{YBa}_{2} \mathrm{Cu}_{3} \mathrm{O}_{7}$, we argue that the superconductivity resides in the $\mathrm{BaO}$ layers. This superconductivity is $s$-wave, not $d$-wave, in the bulk. The trio of ruthenate compounds, doped $\mathrm{Sr}_{2} \mathrm{YRuO}_{6}$, $\mathrm{GdSr}_{2} \mathrm{Cu}_{2} \mathrm{RuO}_{8}$, and $\mathrm{Gd}_{2-z} \mathrm{Ce}_{z} \mathrm{Sr}_{2} \mathrm{Cu}_{2} \mathrm{RuO}_{10}$ all superconduct in their SrO layers, which is why they have almost the same $\sim 49 \mathrm{~K}$ onset temperatures for superconductivity.
\end{abstract}

\section{Faulty evidence for cuprate-plane superconductivity}

Two of the most-cited papers in contemporary physics claim to show that the cuprate-planes of $\mathrm{YBa}_{2} \mathrm{Cu}_{3} \mathrm{O}_{x}$ superconduct $[1,2]$. However, there are problems with both papers that have not been well-recognized: (i) the evidence of superconductivity in the cuprate-planes comes from a jump in charge which was evident in the work of Cava et al., but not in the work of Jorgensen et al.; and (ii) the studies of Jorgensen et al. claim to confirm the data of Cava et al., but actually do not in the most important way: Jorgensen does not have the Cava jump. In fact, a closer examination of the data reveals that only one datum is responsible for the jump in charge that is purportedly evidence for cuprate-plane superconductivity, and this datum was not reproduced in the data of Jorgensen et al. or (to our knowledge) elsewhere. This startling fact has been missed by many people because a continuous line was drawn through rather sparse data and conveyed the impression that the data are much denser than they are. In other words, the concept of cuprate-plane superconductivity has not been confirmed and rests on only one unreproduced datum. (See Fig. 1.)

This is important to realize because the superconducting layers in most (and perhaps all) high-temperature superconductors are not the cuprate-planes, as was implied by the now-infamous fictitious jump in cuprate-plane $\mathrm{Cu}$ charge [3].

Perhaps an independent confirmation of the problem with cuprate-plane superconductivity comes from the scanning tunneling microscopy data on $\mathrm{Bi}_{2} \mathrm{Sr}_{2} \mathrm{CaCu}_{2} \mathrm{O}_{8}$ of the Illinois group [4,5]. (See Fig. 2). The surface layer of this compound, when it is cleaved, is the $\mathrm{BiO}$ layer. Underneath that is the $\mathrm{SrO}$ layer, and then $\mathrm{a} \mathrm{CuO}_{2}$ plane; after that comes a Ca layer and a second $\mathrm{CuO}_{2}$ plane. Apparently the Illinois workers have imaged the $\mathrm{BiO}$ surface layer, and then $\mathrm{CuO}_{2}$ layer that is exposed by a step protruding from the side of the sample, apparently the second $\mathrm{CuO}_{2}$ layer beneath the surface. An examination of their data reveals that their $\mathrm{BiO}$ layer looks like most others, with a U-shaped feature that is indicative of a layer nearby a superconducting layer. But their protruding $\mathrm{CuO}_{2}$ plane does not look at all like a superconductor, and instead of a U-shaped feature, seems to have a band-gap with no density of states in the gap. The Illinois workers have interpreted their observations as evidence of $d$ wave superconductivity in the cuprate-planes, but this interpretation is based primarily on the facts that (i) they assume that the cuprate-planes superconduct, and (ii) faced with the fact that their data do not exhibit the expected U-shape (with a sharper U than BiO's expected for $s$-wave superconductivity), they postulate that they have evidence of $d$-wave superconductivity. But the work of Klemm [6] and Li [7] provides a convincing demonstration that the superconductivity in $\mathrm{Bi}_{2} \mathrm{Sr}_{2} \mathrm{CaCu}_{2} \mathrm{O}_{8}$ is $s$-wave in character, and we have found [8], using the bulk probe of muon spectroscopy, that the superconductivity of $\mathrm{YBa}_{2} \mathrm{Cu}_{3} \mathrm{O}_{7}$ is also $s$-wave in character with an uncertainty of less than $4 \times 10^{-6}$. It is exceedingly unlikely that, in the bulk, $\mathrm{YBa}_{2} \mathrm{Cu}_{3} \mathrm{O}_{7}$ could have $s$ wave pairing, while $\mathrm{Bi}_{2} \mathrm{Sr}_{2} \mathrm{CaCu}_{2} \mathrm{O}_{8}$ has $d$-wave [4] (which cannot be reconciled with other measurements [9]). Hence the better explanation of the facts about the $\mathrm{CuO}_{2}$ layers of the Illinois group is that they feature a band-gap in the $\mathrm{CuO}_{2}$ layers, and those layers do not initiate superconductivity.

\section{Charge transfer in cuprates}

The layer charges of the three layers of $\mathrm{YBa}_{2} \mathrm{Cu}_{3} \mathrm{O}_{x}$, namely the $\mathrm{CuO}$ chain layers, the $\mathrm{BaO}$ layers, and the cuprate-planes (combined with the rare-earth charge) have

*Permanent address. 


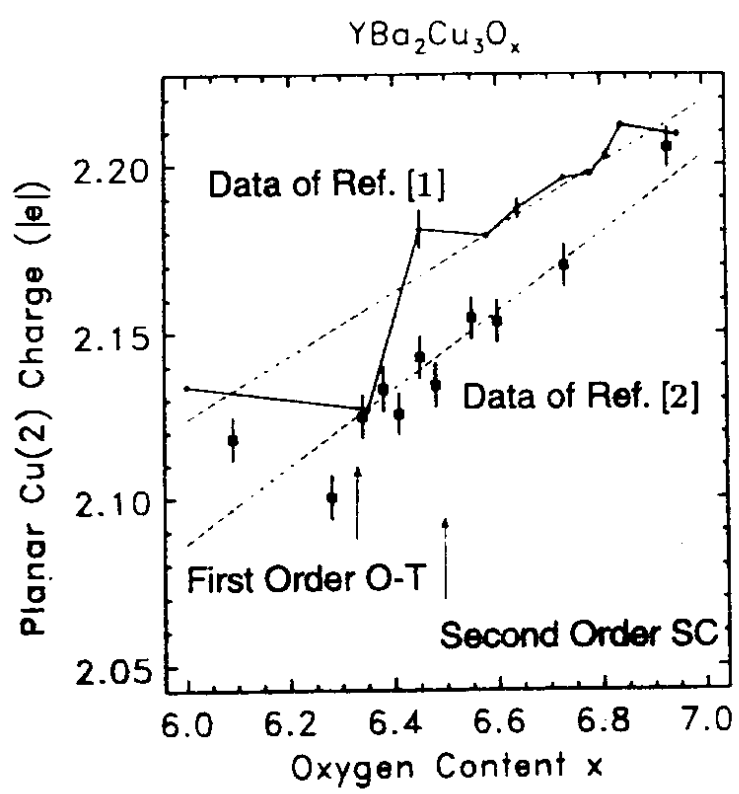

Figure 1. Plot of cuprate-planar $\mathrm{Cu}(2)$ charge in units of $|\mathrm{e}|$ against oxygen content $x$ of $\mathrm{YBa}_{2} \mathrm{Cu}_{3} \mathrm{O}_{x}$, showing the actual data of Ref. [1] and Ref. [2]. Note that in Ref. [1] a smooth line through the data was displayed. Note that the two data sets differ because of a difference in calibration (which should be ignored). For $x>6.4$ both data sets almost define straight lines. Only the single datum near $x=6.4$ from Ref. [1] deviates significantly from a straight line, and the corresponding data of Ref. [2] define a straight line without a jump for $x>6.3$. The jump in the Ref. [1] data, which is non-existent in the data of Ref. [2], is the basis for the argument that the superconductivity is in the cuprate-planes.

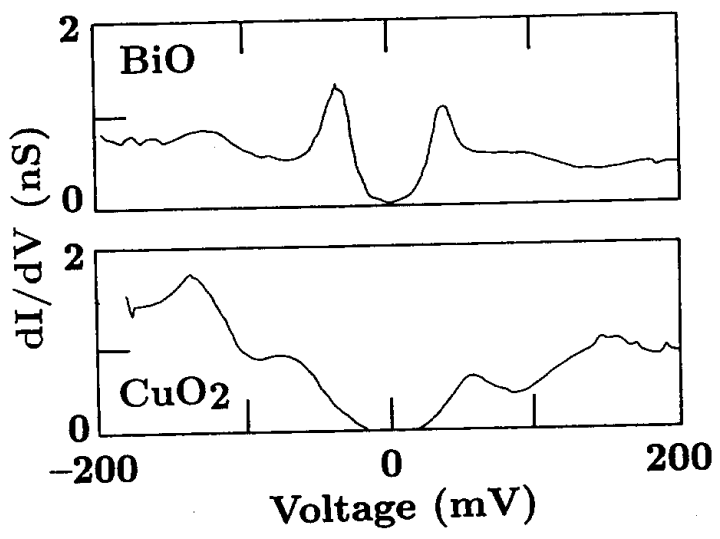

Figure 2. Scanning tunneling microscopy (STM) data of $\mathrm{Bi}_{2} \mathrm{Sr}_{2} \mathrm{CaCu}_{2} \mathrm{O}_{8}$, showing the signal associated with the $\mathrm{BiO}$ layer and with $\mathrm{CuO}_{2}$ plane which protrudes from the sample. Neither layer shows the expected strong peaks of a layer that is exhibiting $s$-wave superconductivity.

been extracted from the data (using the bond-valence-sum method [10]), and have the following features: (1) they are all virtually linear in $x$ and almost the same as the layer charges for $\mathrm{PrBa}_{2} \mathrm{Cu}_{3} \mathrm{O}_{x}$; (2) the $\mathrm{CuO}$ chain layers have $d e$ creasing charge as oxygen content $x$ increases; (3) the $\mathrm{BaO}$ layers and the combined $\mathrm{CuO}_{2} / \mathrm{Rare}$-earth/ $\mathrm{CuO}_{2}$ layers have increasing charge with oxygen content $x$; and (4) only the $\mathrm{BaO}$ layers have charges that appear to change sign as the oxygen content $x$ increases beyond $x=6.4$, and the superconductivity sets in [11]. These facts cause us to assign the superconductivity of $\mathrm{YBa}_{2} \mathrm{Cu}_{3} \mathrm{O}_{7}$ to the $\mathrm{BaO}$ layers.

The same facts also caused us to believe that $\mathrm{PrBa}_{2} \mathrm{Cu}_{3} \mathrm{O}_{7}$ would superconduct [12], which was later show $[13,14]$.

\section{1. $\mathrm{YBa}_{2} \mathrm{Cu}_{3} \mathrm{O}_{7}$ superconductivity: $s$-wave}

We can ask if our data indicate that the superconductivity of $\mathrm{YBa}_{2} \mathrm{Cu}_{3} \mathrm{O}_{7}$ is $s$-wave or $d$-wave in character. Until rather recently, there was a nearly unanimous opinion that the bulk superconductivity is $s$-wave [15-18], but more recently the data have been reinterpreted as having $d$-wave character [19] (although there has been no evidence of a quantitative fit of the $d$-wave theory to the bulk $\mathrm{YBa}_{2} \mathrm{Cu}_{3} \mathrm{O}_{7}$ data [8]).

Figure 3 shows fits to the muon penetration depth extracted from $\mu^{+} \mathrm{SR}$ data and extrapolated to $\mathrm{H}=0$. Those data for $\mathrm{H}=0.05,1.0,3.0$, and 6.0 Tesla were all fit with a single strong-coupling London model $s$-wave curve, after the flux-flow was accounted for. (In earlier 1989 [20] work, which described the data with a similar strong-coupling $s$ wave model, the flux was pinned.) Moreover, the probability that the $d$-wave model fits the data as well a the $s$-wave model was found to be of order one in a million [8]. In the bulk (which is what the muons probe), the superconductivity is definitely $s$-wave in character.

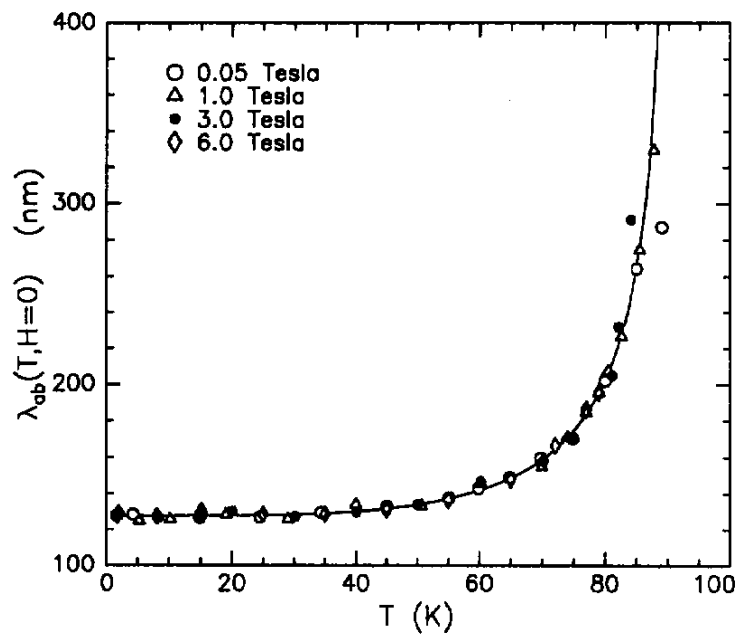

Figure 3. Temperature dependence of the zero-field penetration depth of $\mathrm{YBa}_{2} \mathrm{Cu}_{3} \mathrm{O}_{7}, \lambda_{a b}(\mathrm{~T}, \mathrm{H}=0)$, with the fitted the curve shown is the two-fluid model (which corresponds to strong-coupled $s$ wave pairing), after Ref. [8].

\subsection{Other cuprates than $\mathrm{YBa}_{2} \mathrm{Cu}_{3} \mathrm{O}_{7}$}

Independent evidence indicating that the superconducting layers in the cuprate materials are the $\mathrm{BaO}$ or SrO layers, not the cuprate-planes, is afforded by the $\mathrm{HgBa}_{2} \mathrm{Ca}_{n-1} \mathrm{Cu}_{n} \mathrm{O}_{2 n+2}$ superlattices as functions of $n$, and by the $n=1$ compound of this class as a function of pressure. 
The layer charges behave the same for the superlattices versus $n$ and for the $n=1$ compound versus pressure $p$ [21-23]: The charges of the $\mathrm{BaO}$ layers increase with increasing number of layers $n$ and pressure $p$, while the $\mathrm{Hg}$ layer-charges remain constant, and the charges of the cuprate-planes decrease. Since $\mathrm{T}_{c}$ increases with the number of layers $n$ and with pressure $p$, the superconductivity must originate in the $\mathrm{BaO}$ layers (whose charges also increase), not in the cuprateplanes (whose charges decrease) [24].

\section{$3 \quad \mathrm{Sr}_{2} \mathbf{Y R u O}_{6}$ doped with $\mathrm{Cu}$}

$\mathrm{Sr}_{2} \mathrm{YRuO}_{6}$ is an interesting compound because, upon doping with $\mathrm{Cu}$, it begins to superconduct around $\sim 49 \mathrm{~K}$ and becomes fully superconducting at $\sim 23 \mathrm{~K}[25]$. It also has two superconducting sister compounds that contain cuprate-planes, which superconduct slightly below about $\sim 49 \mathrm{~K}$ [25]: $\mathrm{GdSr}_{2} \mathrm{Cu}_{2} \mathrm{RuO}_{8}$ and $\mathrm{Gd}_{2-z} \mathrm{Ce}_{z} \mathrm{Sr}_{2} \mathrm{Cu}_{2} \mathrm{RuO}_{10}$ [26]. The near coincidence of the onset temperatures for superconductivity in the three ruthenate compounds, which we term the O6's (as in $\mathrm{Cu}$-doped $\mathrm{Sr}_{2} \mathrm{YRuO}_{6}$ ), the O8's (as in $\mathrm{GdSr}_{2} \mathrm{Cu}_{2} \mathrm{RuO}_{8}$ ), and the O10's (as in $\mathrm{Gd}_{2-z} \mathrm{Ce}_{z} \mathrm{Sr}_{2} \mathrm{Cu}_{2} \mathrm{RuO}_{10}$ ), makes this class of three types of compounds especially worthwhile to investigate.

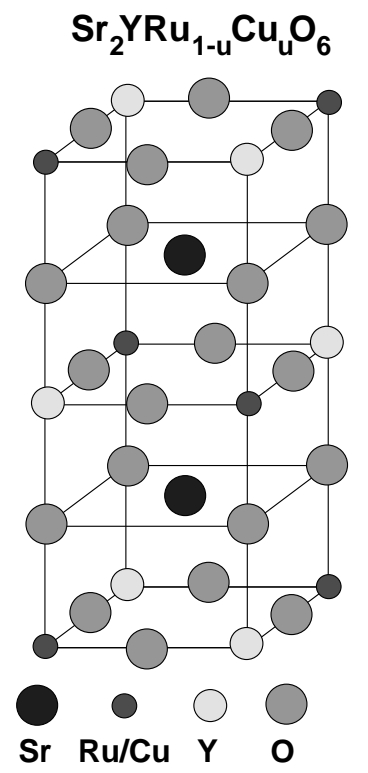

Figure 4. Crystal structure of $\mathrm{Sr}_{2} \mathrm{YRu}_{1-u} \mathrm{Cu}_{u} \mathrm{O}_{6}$.

The simplest of these compounds is $\mathrm{Sr}_{2} \mathrm{YRuO}_{6}$ which is a two-layer compound with each pair of $(\mathrm{SrO})_{2}$ layers having a $\mathrm{YRuO}_{4}$ layer in between. (See Fig. 4). Since this compound has only two kinds of layers, it is rather straightforward to select the superconducting layer: namely the $\mathrm{SrO}$ layer, which is the one without a strong magnetic field, and the one analogous to $\mathrm{BaO}$. The $\mathrm{YRuO}_{4}$ layer is ferromagnetic in its $a-b$ plane, with its magnetic moments stacked antiferromagnetically along the $c$-axis, and oriented in the $\pm(1,1)$ directions of the $a-b$ plane. Muons in this material stop at one of two nearly identical sites: (i) the $\mu_{\mathrm{O}(1,2)}$ site which is actually two sites (due to the difference between $\mathrm{Y}$ and $\mathrm{Ru}$ ) near the center of the $\mathrm{YRuO}_{4}$ layer; and (ii) the $\mu_{\mathrm{O}(3)}$ site, which is about midway between two oxygen ions on the edge of a SrO plane. Clearly the $\mathrm{YRuO}_{4}$ layer is highly magnetic (and hence rather hostile to superconductivity), while the SrO layer has an average magnetic field of zero and is the locus of superconductivity.

The muon data for the SrO-plane site $\left(\mu_{\mathrm{O}(3)}\right)$ show a time-dependence that reveals (i) an onset of superconductivity near $\sim 49 \mathrm{~K}$ [25], (ii) the onset of spin-glass behavior at $\approx 29.3 \mathrm{~K}$ [25], and (iii) the onset of diamagnetism at lower temperatures [25]. Complementing the muon data are resistivity data, which show that the resistance vanishes at $\mathrm{T}_{c} \approx 23 \mathrm{~K}$ [25], at which temperature the superconductivity becomes complete. Clearly $\mathrm{Sr}_{2} \mathrm{YRu}_{1-u} \mathrm{Cu}_{u} \mathrm{O}_{6}$ is a superconductor, whose superconductivity originates at $\sim 49 \mathrm{~K}$ and becomes complete at $\sim 23 \mathrm{~K}$ when the $\mathrm{Ru}$ librations turn off.

Doped $\mathrm{Sr}_{2} \mathrm{YRuO}_{6}$ has a sister compound with $\mathrm{Gd}^{+3}$, $\mathrm{Cu}$-doped $\mathrm{Ba}_{2} \mathrm{GdRuO}_{6}$, which does not superconduct. We attribute its non-superconductivity to the pair-breaking by $\mathrm{Gd}^{+3}$, which has $\mathrm{L}=0$ and $\mathrm{J} \neq 0$, and breaks Cooper pairs located in the adjacent $\mathrm{SrO}$ layers. Hence doped $\mathrm{Ba}_{2} \mathrm{GdRuO}_{6}$ does not superconduct [27], but its sister compound, doped $\mathrm{Sr}_{2} \mathrm{YRuO}_{6}$, does superconduct in its $\mathrm{SrO}$ layers. For similar reasons, $\mathrm{Gd}_{2-z} \mathrm{Ce}_{z} \mathrm{CuO}_{4}$ does not superconduct, but many other (Rare-earth) ${ }_{2-z} \mathrm{Ce}_{z} \mathrm{CuO}_{4}$ compounds do superconduct.

\section{$4 \mathrm{GdSr}_{2} \mathrm{Cu}_{2} \mathrm{RuO}_{8}$ and $\mathbf{G d}_{2-z} \mathbf{C e}_{z} \mathbf{S r}_{2} \mathbf{C u}_{2} \mathbf{R u O}_{10}$}

$\mathrm{Sr}_{2} \mathrm{YRu}_{1-u} \mathrm{Cu}_{u} \mathrm{O}_{6}$ has two superconducting sister compounds, $\mathrm{GdSr}_{2} \mathrm{Cu}_{2} \mathrm{RuO}_{8}$ and $\mathrm{Gd}_{2-z} \mathrm{Ce}_{z} \mathrm{Sr}_{2} \mathrm{Cu}_{2} \mathrm{RuO}_{10}$. They superconduct at nearly the $\sim 49 \mathrm{~K}$ onset temperature for $\mathrm{Sr}_{2} \mathrm{YRu}_{1-u} \mathrm{Cu}_{u} \mathrm{O}_{6}$ 's superconductivity - raising the possibility that the superconductivity of all three materials originates in the same physics [26].

Of course this raises the question of what roles do the cuprate-planes have in the superconductivity of these compounds? Obviously the answer is "none" for doped $\mathrm{Sr}_{2} \mathrm{YRuO}_{6}$, which has no cuprate-planes and fewer than $1 \%$ $\mathrm{Cu}$ atoms even capable of participating in any cuprate-planelike behavior. This, of course, raises the question of whether the cuprate-planes even superconduct in these other materials, $\mathrm{GdSr}_{2} \mathrm{Cu}_{2} \mathrm{RuO}_{8}$ and $\mathrm{Gd}_{2-z} \mathrm{Ce}_{z} \mathrm{Sr}_{2} \mathrm{Cu}_{2} \mathrm{RuO}_{10}$. (We shall see that they do not).

First we examine $\mathrm{GdSr}_{2} \mathrm{Cu}_{2} \mathrm{RuO}_{8}$, which does superconduct. Do its cuprate-planes superconduct? Or do its $\mathrm{SrO}$ layers superconduct? Or both? 
To address these questions, we analyze magnetic resonance data taken [28] on $\mathrm{GdSr}_{2} \mathrm{Cu}_{2} \mathrm{RuO}_{8}$ at $\mathrm{T}=120 \mathrm{~K}$ (above $\mathrm{T}_{c} \approx 49 \mathrm{~K}$ ) at a frequency of $20 \mathrm{GHz}$, and with fields $\mathbf{H}_{r f} \perp \mathbf{H}_{d c}$. $\left(\mathbf{H}_{r f}\right.$ is the radio-frequency field; we also refer to the direct-current field $\mathbf{H}_{d c}$ as $\mathbf{H}$ ). These data can be decomposed into two peaks, one associated with the Gd electron spin resonance (ESR), and the other associated with a $\mathrm{Cu}$ signal which is either an anti-ferromagnetic resonance or a weak ferromagnetic resonance (the equipment does not determine which) [28]. See Fig. 5 [29]. Our point is that, if the $\mathrm{Cu}$ in this compound is magnetic and resonating, the cuprate-planes almost certainly do not superconduct.

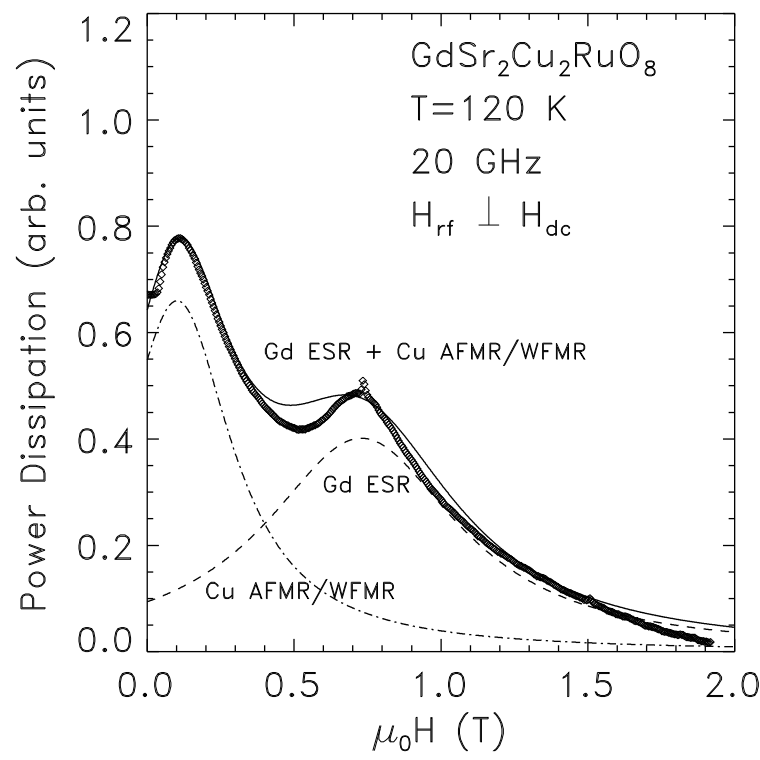

Figure 5. Power dissipation (in arbitrary units) in $\mathrm{GdSr}_{2} \mathrm{Cu}_{2} \mathrm{RuO}_{8}$ versus applied magnetic field $\mu_{0} \mathrm{H}$ in Tesla for $\mathbf{H}_{r f} \perp \mathbf{H}_{d c}$, where $\mathbf{H}_{d c}=\mathbf{H}$. The (i) heavy line, (ii) the dashed curve, and (iii) the dashed-dotted curve are (i) the data, (ii) the Gd electron spin resonance, and (iii) the $\mathrm{Cu}$ weak ferromagnetic resonance or antiferromagnetic resonance. The latter two curves add up to the thin solid line. The data were taken at $20 \mathrm{GHz}$ and at a temperature of $120 \mathrm{~K}$ (well above $\mathrm{T}_{c}$ ). We attribute the broad low-field signal to $\mathrm{Cu}$, although it has been assigned to $\mathrm{Ru}$ by other authors [29].

There should be no dispute over the well-known Gd ESR peak, so we shall discuss the identification of the "Cu signal" primarily. The signal we term the " $\mathrm{Cu}$ signal" is certainly due to $\mathrm{Cu}$ and not due to $\mathrm{Ru}$ (the only other possible magnetic ion that could resonate in $\mathrm{GdSr}_{2} \mathrm{Cu}_{2} \mathrm{RuO}_{8}$ ). A similar $\mathrm{Cu}$ signal is observed in $\mathrm{GdSr}_{2} \mathrm{Cu}_{2} \mathrm{NbO}_{8}$, which has no $\mathrm{Ru}$ (and does not superconduct). In that material, the resonance must be associated with $\mathrm{Cu}$, since the Gd resonance is identified and there is no other magnetic ion.

The "Cu signal" is $\mathrm{Gd}_{2-z} \mathrm{Ce}_{z}$ in $\mathrm{GdSr}_{2} \mathrm{Cu}_{2} \mathrm{RuO}_{10}$ [28], and persists up to above $150 \mathrm{~K}$ although $\mathrm{Ru}$ does not give a magnetic resonance above $\sim 133 \mathrm{~K}$, which mean that the "Cu signal" is due to $\mathrm{Cu}$, not $\mathrm{Ru}$.

To strengthen this argument, we have studied nine related compounds containing $\mathrm{Ru}$, and we see no $\mathrm{Ru}$ magnetic resonance in any of them: these include the superconductors $\mathrm{Gd}_{1.5} \mathrm{Ce}_{0.5} \mathrm{Sr}_{2} \mathrm{Cu}_{2} \mathrm{RuO}_{10}, \mathrm{Eu}_{1.5} \mathrm{Ce}_{0.5} \mathrm{Sr}_{2} \mathrm{Cu}_{2} \mathrm{RuO}_{10}$,
$\mathrm{GdSr}_{2} \mathrm{Cu}_{2} \mathrm{RuO}_{8}, \mathrm{EuSr}_{2} \mathrm{Cu}_{2} \mathrm{RuO}_{8}$, and $\mathrm{Sr}_{2} \mathrm{YRu}_{1-u} \mathrm{Cu}_{u} \mathrm{O}_{6}$, and the magnetically-ordered non-superconductors $\mathrm{SrRuO}_{3}$, $\mathrm{Sr}_{3} \mathrm{Ru}_{2} \mathrm{O}_{7}, \mathrm{GdSr}_{2} \mathrm{Cu}_{2} \mathrm{NbO}_{8}$, and $\mathrm{Ba}_{2} \mathrm{GdRu}_{1-u} \mathrm{Cu}_{u} \mathrm{O}_{6}$ [30]. $\mathrm{Gd}_{2-z} \mathrm{Ce}_{z} \mathrm{Sr}_{2} \mathrm{Cu}_{2} \mathrm{RuO}_{10}$ behaves similarly to $\mathrm{GdSr}_{2} \mathrm{Cu}_{2} \mathrm{RuO}_{8}$, and also superconducts in its SrO layers, not in its cuprate-planes [28].

\section{Summary}

We have challenged the widely held notion that the cuprateplanes are the carriers of high-temperature superconductivity, and have instead proposed that the superconducting elements of most superconductors are not the cuprate-planes, but instead the $\mathrm{BaO}$ or $\mathrm{SrO}$ layers. (Some materials may also involve interstitial oxygen [31], which we have not discussed here.)

We have examined the three materials, doped $\mathrm{Sr}_{2} \mathrm{YRuO}_{6}, \mathrm{GdSr}_{2} \mathrm{Cu}_{2} \mathrm{RuO}_{8}$, and $\mathrm{Gd}_{2-z} \mathrm{Ce}_{z} \mathrm{Sr}_{2} \mathrm{Cu}_{2} \mathrm{RuO}_{10}$, and we have found extremely powerful evidence that all three superconduct in their SrO layers, and not in their cuprate-planes.

A complete microscopic theory of high-temperature superconductivity will be presented elsewhere.

\section{Acknowledgments}

We are grateful to the U. S. Office of Naval Research for their financial support (Contract No. N00014-03-1-0375).

\section{References}

[1] R. J. Cava, A. W. Hewat, E. A. Hewat, B. Batlogg, M. Marezio, K. M. Rabe, J. J. Krajewski, W. F. Peck, Jr., and L. W. Rupp, Jr., Physica C, 165, 419 (1990); R. Cava, Synthesis and crystal chemistry of high- $T_{c}$ oxide superconductors, in Processing and Properties of High- $T_{c}$ Superconductors, Vol. 1, edited by S. Jin (World Scientific, Singapore, 1993), p. 1 et seq., especially p. 12.

[2] J. D. Jorgensen, B. W. Veal, A. P. Paulikas, L. J. Nowicki, G. W. Crabtree, H. Claus, and W. K. Kwok, Phys. Rev. B 41, 1863 (1990); J. D. Jorgensen, Phys. Today, 34 (June, 1991).

[3] In some superconductors, such as $\mathrm{HgBa}_{2} \mathrm{Cu}_{n-1} \mathrm{Cu}_{n} \mathrm{O}_{2 n+2}$, the holes are clearly accumulating in the $\mathrm{BaO}$ layers rather than in the cuprate planes. See H. A. Blackstead and J. D. Dow, Solid State Commun. 95, 613 (1995).

[4] S. Misra, S. Oh, D. J. Hornbaker, T. DiLuccio, J. N. Eckstein, and A. Yazdani, Phys. Rev. Lett. 89, 087002 (2002).

[5] Misra et al. claim to image a cuprate-plane protruding from the side of a superconductor. For a discussion of scanning tunneling microscopy of the surfaces of bulk superconductors, see S. H. Pan, J. P. O’Neal, R. L. Badzey, C. Chamon, H. Ding, J. R. Engelbrecht, Z. Wang, H. Eisaki, S. Uchida, A. K. Gupta, K.-W. Ng, E. W. Hudson, K. M. Lang, and J. C. Davis, Nature 413, 282 (2001).

[6] R. A. Klemm, C. T. Rieck and K. Scharnberg, Phys. Rev. B61, 5913 (2000); R. A. Klemm, Intl. J. Mod. Phys. B12, 2920 (1998), and references therein. 
[7] Q. Li, Y. N. Tsay, M. Suenaga, R. A. Klemm, G. D. Gu, and N. Koshizuka, Phys. Rev. Lett. 83, 4160 (1999); Q. Li, Y. N. Tsay, M. Suenaga, G. D. Gu, and N. Koshizuka, Physica C282-287, 1495 (1997).

[8] D. R. Harshman, W. J. Kossler, X. Wan, A. T. Fiory, A. J. Greer, D. R. Noakes, C. E. Stronach, E. Koster, A. Erb, and J. D. Dow, "Nodeless pairing state in single-crystal $\mathrm{YBa}_{2} \mathrm{Cu}_{3} \mathrm{O}_{7}$." in press.

[9] D. R. Harshman, R. N. Kleiman, M. Inui, G. P. Espinosa, D. B. Mitzi, A. Kapitulnik, T. Pfiz, and D. L. Williams, Phys. Rev. Lett. 67, 3152 (1991).

[10] I. D. Brown and D. Altermatt, Acta Crystallogr., sect. B: Struct. Sci. B41, 244 (1985); D. Altermatt and I. D. Brown, Acta Crystallogr., Sect. B: Struct. Sci. B41, 241 (1985); I. D. Brown, J. Solid State Chem. 82, 122 (1989); I. D. Brown and K. K. Wu, Acta Crystallogr., Sect. B: Struct. Crystallogr. Cryst. Chem. B32, 1957 (1976); I. D. Brown, Structure and Bonding in Crystals, Vol. II, edited by M. O'Keefe and A. Navrotsky, pp. 1-20 (Academic Press, New York, 1980).

[11] The charges on the various layers are given in J. D. Dow, H. A. Blackstead, and D. R. Harshman, Physica C364-365, 74 (2001).

[12] H. A. Blackstead and J. D. Dow, Phys. Rev. B51, 11830 (1995).

[13] H. A. Blackstead, D. B. Chrisey, J. D. Dow, J. S. Horwitz, A. E. Klunzinger, and D. B. Pulling, Phys. Lett. A207, 109 (1995).

[14] H. A. Blackstead, J. D. Dow, D. B. Chrisey, J. S. Horwitz, P. J. McGinn, M. A. Black, A. E. Klunzinger, and D. B. Pulling, Phys. Rev. B54, 6122 (1996).

[15] D. R. Harshman, G. Aeppli, E. J. Ansaldo, B. Batlogg, J. H. Brewer, J. F. Carolan, R. J. Cava, M. Celio, A. C. D. Chaklader, W. N. Hardy, S. R. Kreitzman, G. M. Luke, D. R. Noakes, and M. Senba, Phys. Rev. B 36, 2386 (1987).

[16] B. Pümpin, H. Keller, W. Kündig, W. Odermatt, I. M. Savic, J. W. Schneider, H. Simmler, P. Zimmermann, J. G. Bednorz, Y. Maeno, K. A. Müller, C. Rossel, E. Kaldis, S. Rusiecki, W. Assmus, and J. Kowalewski, Physica C162-164, 151 (1989).

[17] B. Pümpin, H. Keller, W. Kündig, W. Odermatt, I. M. Savic, J. W. Schneider, H. Simmler, P. Zimmermann, E. Kaldis, S. Rusiecki, Y. Maeno, and C. Rossel, Phys. Rev. B 42, 8019 (1990).

[18] A. G. Sun, D. A. Cajewski, M. B. Maple, and R. C. Dynes, Phys. Rev. Lett. 72, 2267 (1994).
[19] J. E. Sonier, J. H. Brewer, and R. F. Kiefl, Rev. Mod. Phys. 72, 769 (2000).

[20] D. R. Harshman, L. F. Schneemeyer, J. V. Waszczak, G. Aeppli, R. J. Cava, B. Batlogg, L. W. Rupp, E. J. Ansaldo, and D. Ll. Williams, Phys. Rev. B 39, 851 (1989).

[21] H. A. Blackstead and J. D. Dow, Solid State Commun. 95, 613 (1995).

[22] J. D. Dow and D. R. Harshman, J. Phys. Chem. Solids 63, 2309 (2002).

[23] J. D. Dow and D. R. Harshman, Phil. Mag. B82, 1055 (2002).

[24] For a variety of opposing views, most relying on surfacesensitive experiments, see D. A. Wollman, D. J. Van Harlingen, J. Giapintzakis, and D. M. Ginsberg, Phys. Rev. Lett. 74, 797 (1995); A. Lanzara, P. V. Bogdanov, X. J. Zhou, S. A. Kellar, D. L. Feng, E. D. Lu, T. Yoshida, H. Eisaki, A. Fujimori, K. Kishio, J.-I. Shimoyama, T. Noda, S. Uchida, Z. Hussain, and Z. X. Shen, Nature 412, 510 (2001); and J. R. Kirtley, C. C. Tsuei, K. A. Moler, J. Z. Sun, A. Gupta, Z. F. Ren, J. H. Wang, Z. Z. Li, H. Raffy, J. Mannhart, H. Hilgenkamp, B. Mayer, and Ch. Gerber, Czech. J. Phys. 46, Suppl. S6, 3169 (1996), and subsequent work.

[25] D. R. Harshman, W. J. Kossler, A. J. Greer, D. R. Noakes, C. E. Stronach, E. Koster, M. K. Wu, F. Z. Chien, J. P. Franck, I. Isaac, and J. D. Dow, Phys. Rev. B67, 054509 (2003).

[26] D. R. Harshman, J. D. Dow, W. J. Kossler, D. R. Noakes, C. E. Stronach, A. J. Greer, E. Koster, Z. F. Ren, and D. Z. Wang, Muon spin rotation study of $\mu \mathrm{SR}$ of $\mathrm{GdSr}_{2} \mathrm{Cu}_{2} \mathrm{RuO}_{8}$. Phil Mag., Submitted.

[27] D. R. Harshman, W. J. Koessler, A. J. Greer, C. E. Stronach, D. R. Noakes, E. Koster, M. K. Wu F. Z. Chien, H. A. Blackstead D. B. Pulling, and J. D. Dow, Physica C364-365, 392 (2001).

[28] J. D. Dow, H. A. Blackstead, Z. F. Ren, and D. Z. Wang, Magnetic resonance of $\mathrm{Cu}$ and of $\mathrm{Gd}$ in insulating $\mathrm{GdSr}_{2} \mathrm{Cu}_{2} \mathrm{NbO}_{8}$ and in superconducting $\mathrm{GdSr}_{2} \mathrm{Cu}_{2} \mathrm{RuO}_{8}$. Submitted.

[29] A. Fainstein, E. Winkler, A. Butera, and J. Tallon, Phys. Rev. B 60, 12597 (1999).

[30] H. A. Blackstead, J. D. Dow, et al., "Magnetically ordered copper in superconducting rutheno-cuprates: evidence against spin-fluctuation models of high-temperature superconductivity," in preparation.

[31] J. D. Dow and M. Lehmann, Phil. Mag. 83, 527 (2003). 